\title{
Trans-membrane Fluorescence Enhancement by Carbon Dots: Ionic Interactions and Energy Transfer
}

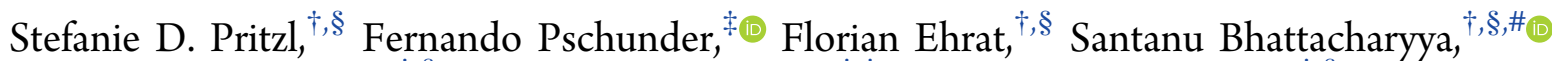

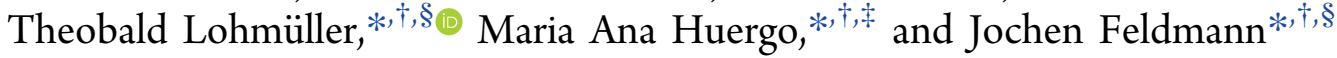

${ }^{\dagger}$ Chair for Photonics and Optoelectronics, Nano-Institute Munich, Department of Physics, Ludwig-Maximilians-Universität (LMU), Königinstraße 10, 80539 Munich, Germany

${ }^{\S}$ Nanosystems Initiative Munich (NIM) and Center for Nanoscience (CeNS), Schellingstraße 4, 80799 Munich, Germany

${ }^{\ddagger}$ Instituto de Investigaciones Fisicoquímicas Teóricas y Aplicadas (INIFTA), Universidad Nacional de La Plata-CONICET, Sucursal 4 Casilla de Correo 16, 1900 La Plata, Argentina

Supporting Information

ABSTRACT: We report on trans-membrane interactions between blueemitting carbon dots (CDs) and fluorescein. Hydrophobic CDs with a positive surface charge are embedded as-synthesized in the lipophilic sheet of the bilayer membrane of large synthetic phospholipid vesicles. The vesicles are prepared by mixing DOPC phospholipids and lipid molecules that contain anionic fluorescein attached to their hydrophilic head. Due to attractive electrostatic interactions, the $\mathrm{CDs}$ and fluorescein conjoin within the vesicle membrane, which leads to photoluminescence enhancement of fluorescein and facilitates trans-membrane energy transfer between the CDs and the dye.

KEYWORDS: Membrane sensing, carbon dots, lipid bilayer membranes, trans-membrane energy transfer, ionic interactions

$\mathrm{T}$ he plasma membrane of living cells is semipermeable for gases and small molecules, while ions can only selectively pass the membrane barrier via special channel proteins and ion pumps. ${ }^{1}$ This tight regulation is required to establish an electrical trans-membrane potential that has an important biological function. The ion exchange between the cell interior and exterior, for example, results in membrane depolarization that is causing the excitation of neurons or muscle fibers. The standard technique for measuring such ion-transport or membrane potential changes is "patch-clamp", where a single cell or a lipid vesicle is "patched" with a glass micropipette ${ }^{2}$ or with a porous glass slide in a planar configuration. ${ }^{3}$ The reliability and robustness of patch-clamp for electrophysiological measurements are without question. Experimentally, however, there are some challenges. For example, a patchclamp measurement requires a physical contact and is typically less suitable for investigations of larger cellular networks or neuronal circuits.

Voltage-sensing dyes that change their fluorescence intensity in response to an external electric field or to a change of the membrane potential are an alternative approach to study membrane properties by optical means. ${ }^{4}$ In their pioneering work, Tsien and co-workers ${ }^{5}$ studied Förster resonance energy transfer (FRET) between donor-fluorophores in the membrane of living cells and negatively charged acceptor fluorophores in solution. They observed efficient energy transfer only for a negative membrane potential, when the anionic fluorophores were located next to the donor molecules on the membrane surface. Upon membrane depolarization, the acceptor molecules translocated into the bilayer, which increased the separation distance between donor and acceptor and caused the energy transfer to be less efficient.

FRET is a powerful approach for studying lipid membranes that has been widely used for investigating the lateral distribution and interactions of membrane proteins and lipids. ${ }^{6}$ However, trans-membrane FRET processes as observed by Tsien et al., where the donor and acceptor are separated in different bilayer leaflets, are rarely explored. Furthermore, many fluorescent proteins and dye molecules are sensitive to photobleaching ${ }^{7}$ and can unintentionally integrate into the membrane. ${ }^{5,8}$ In this case, the membrane potential is perturbed or the FRET efficiency could change. ${ }^{9,10}$

Such issues can be avoided by using inorganic semiconductor nanocrystals or quantum dots (QDs) for membrane labeling. QDs distinguish themselves from most organic fluorophores by a high quantum yield and photochemical stability. ${ }^{11}$ Kloepfer et al. have shown that FRET between membrane embedded QDs and water-soluble dyes in solution can be used for monitoring neuronal network dynamics in large areas and on slow time scales. ${ }^{12}$ Semiconductor nanorods that span the bilayer membrane were also applied to detect the formation of action potentials in excitable cells by taking advantage of the quantum confined Stark effect ${ }^{13,14}$ and Auger recombination. ${ }^{15}$ However, when embedded in a lipid

Received: March 14, 2019

Revised: $\quad$ May 2, 2019

Published: May 3, 2019 
membrane, QD stability can be a problem. Zheng et al. found, for example, that CdSe-QDs that are located in the inner hydrophobic sheet of a fluid lipid bilayer membrane are prone to corrosion and deterioration. ${ }^{16}$ This is unfavorable for many biological applications, particularly because QDs are often synthesized out of toxic elements. ${ }^{17}$

Here, biocompatible fluorescent carbon dots (CDs) fill a missing gap. ${ }^{18,19} \mathrm{CDs}$ are built up from polycyclic aromatic hydrocarbon domains ${ }^{20}$ and small molecular fluorophores ${ }^{21}$ and display unique tunable, multicolor photoluminescence properties and photostability. ${ }^{22}$ At the same time, their chemical stability and small size as well as their two-photon activity render them to be ideal candidates for biological applications. ${ }^{22-24} \mathrm{CD}$ s have shown to be excellent membrane markers for both synthetic lipid membranes and the membranes of living cells. ${ }^{25-27}$ So far, FRET between CDconjugates and dye-labeled lipid molecules has only been reported for samples where both donor and acceptor were embedded in the same leaflet of a bilayer membrane. ${ }^{27,28}$ However, trans-membrane energy transfer, where CDs and dye molecules are located in different bilayer regions, has not been investigated, yet.

In this Letter, we demonstrate a strategy to incorporate hydrophobic blue-emitting CDs with a positive surface charge between the two leaflets of a DOPC (1,2-dioleoyl-sn-glycero-3phosphocholine) phospholipid bilayer membrane (Figure 1).

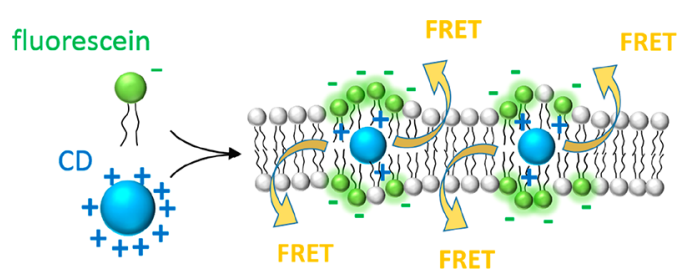

Figure 1. Schematic of the $\mathrm{CD} / \mathrm{F}$ configuration in a bilayer membrane: Phospholipid molecules that are labeled with negatively charged fluorescein (PE CF) are mixed with DOPC phospholipids (gray) and positively charged blue emitting $\mathrm{CDs}$ to self-assemble into a bilayer membrane. The hydrophobic CDs are located close to the lipid tails between the bilayer leaflets, while the fluorescein molecules are covalently bound to the hydrophilic lipid head-groups. Both fluorescein and the CD form a FRET pair that is colocalized within the membrane sheet due to attractive electrostatic interactions.

In addition, fluorescein-labeled phospholipid molecules (PECF-1,2-dioleoyl-sn-glycero-3-phosphoethanolamine- $N$ (carboxyfluorescein)) with a negative charge, were added to the sample. In these lipids, the fluorescein (F) is covalently bound to the hydrophilic headgroup, which prevents the dye from integrating into the membrane center (Supporting Information, Figure S1). The CDs and the fluorescein are therefore separated in different bilayer regions. We demonstrate that electrostatic interactions between the CDs and the dye molecules lead to an increase of emission intensity. Furthermore, we show that CDs and fluorescein form a FRET pair across the bilayer membrane with the CDs being the donor and fluorescein the acceptor. Controlling transmembrane energy transfer as shown with the $\mathrm{CD} /$ fluorescein system offers a promising route for efficient and biocompatible implementations in voltage sensing strategies and imaging applications.

Hydrophobic CDs are synthesized from ascorbic acid and hexadecylamine (HDA) according to the protocol by Cheng et al. $^{29}$ The size of $\sim 1.0-1.5 \mathrm{~nm}$ of the spherical CDs was determined by atomic force microscopy and dynamic light scattering (Supporting Information, Figure S3). Large lipid vesicles $(d=5-10 \mu \mathrm{m})$ are then prepared by mixing either CDs or fluorescein $(F)$ or both components with DOPC phospholipids in a HEPES buffer solution $(\mathrm{pH} \approx 7.1)$. In aqueous solution, the hydrophobic CDs are embedded between the carbon hydrate tails of the lipid molecules. The fluorescein dyes, at the same time, are located in the hydrophilic side of the membrane sheets (Figure 1).

The combination of CDs and fluorescein was chosen for several reasons: First, the $\mathrm{CD}$ fluorescence and fluorescein absorption display a large spectral overlap, which is a requirement for FRET. ${ }^{30}$ Photoluminescence (PL) and absorption measurements of vesicles that contain either CDs or fluorescein are shown in Figure 2. When excited at $360 \mathrm{~nm}$,

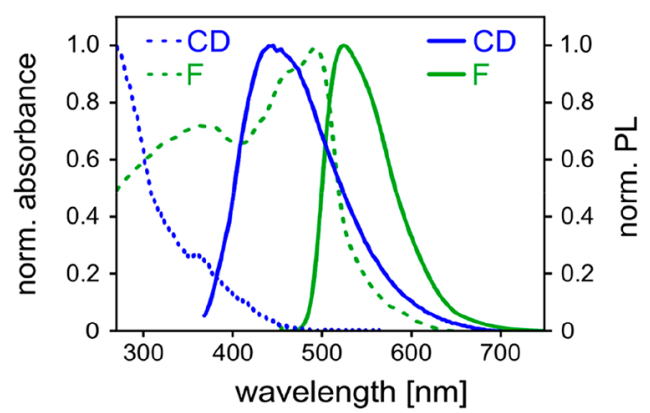

Figure 2. PL and absorption spectra of CDs and fluorescein: The CD absorption spectrum (blue dotted line) shows a small peak at $~ 360$ $\mathrm{nm}$. CD absorption sets at $\sim 450 \mathrm{~nm}$. The PL spectrum of the carbon dots (blue line) and the absorbance of the fluorescein (green dotted line) show a spectral overlap, which is essential for energy transfer from donor to acceptor in a FRET process. (For comparison, PL spectra of CDs in HEPES buffer and $\mathrm{ddH}_{2} \mathrm{O}$ are also shown as Supporting Information, Figure S2).

the PL spectrum of the CD-vesicles shows a maximum at 450 $\mathrm{nm}$, while the UV-vis absorption spectrum of fluorescein features a broad resonance around 410-520 nm. The PL spectrum of fluorescein acquired under $450 \mathrm{~nm}$ excitation shows a maximum at $530 \mathrm{~nm}$. Second, the CDs and fluorescein are a well suited system for FRET due to attractive electrostatic interactions between both partners. The vesicles are prepared in a HEPES buffer solution at a $\mathrm{pH}$ of 7.1. At this $\mathrm{pH}$, fluorescein occurs in a monoanion form. ${ }^{31} \mathrm{CDs}$ can have a positive or negative surface charge, depending on the preparation conditions. $^{21}$ In the experiment reported here, the addition of HDA during the CD synthesis results in a positive surface charge with a $\mathrm{CD}$ zeta-potential of $+60 \mathrm{mV}$. The opposing charges of the CDs and fluorescein are advantageous, as they keep both partners close together. In the bilayer membrane, the fluorescein dyes and the CDs are likely attracted to each other, even though the bilayer itself is in principle a dynamic environment where individual lipid molecules display a certain degree of lateral mobility. ${ }^{32}$ Nevertheless, in fluorescence microscopy the fluorescein, $\mathrm{CD}$, and $\mathrm{CD} / \mathrm{F}$ vesicles all show a homogeneous coloring, indicating an even distribution of the fluorescent emitters in the vesicle membrane and no indication of domain formation or large particle aggregates (Figure $3 \mathrm{a}-\mathrm{c}$ ). Third, the Förster distance for energy transfer to fluorescein is in a range of 3-5 $\mathrm{nm},{ }^{9,10,33,34}$ while the width of a DOPC bilayer membrane in 


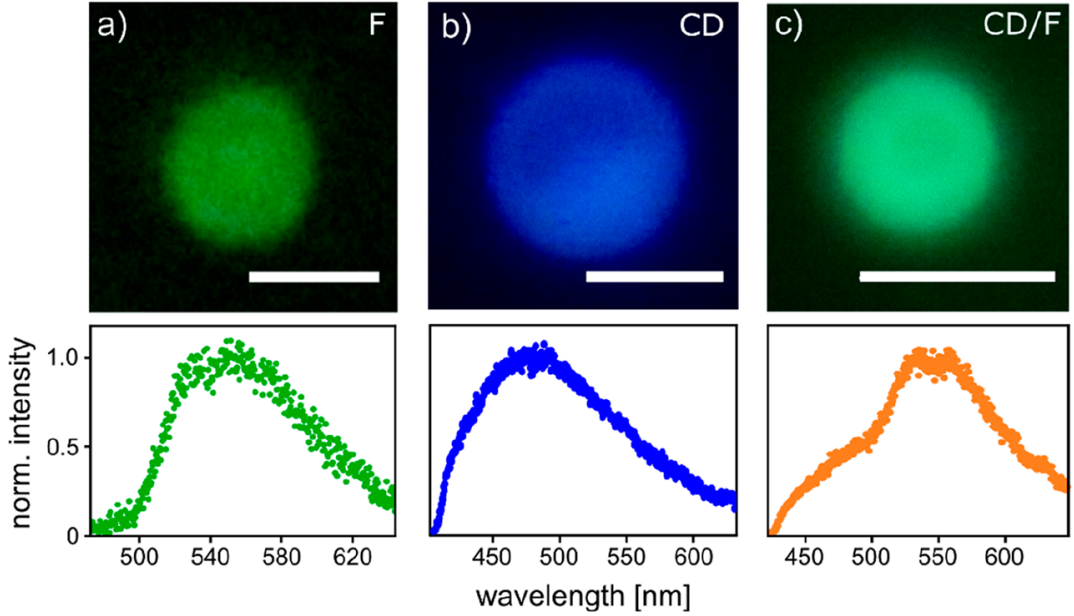

Figure 3. Fluorescence microscopy images and corresponding PL spectra of fluoresceine (F), carbon dot (CD), and CD/F labeled lipid vesicles: Fluorescein labeled vesicles show a characteristic green fluorescence (a), while the CD labeled vesicles display blue emission (b). The CD/F vesicles display a turquoise color upon excitation with $360 \mathrm{~nm}$ due to the presence of both emitters. The corresponding normalized fluorescence spectra of each vesicle are shown below the images. Scale bars: $10 \mu \mathrm{m}$.

aqueous solution is $\sim 6 \mathrm{~nm} .{ }^{35}$ The distance between the donor $\mathrm{CDs}$ in the center of the bilayer and the acceptor fluorescein on the membrane surface is therefore around $3 \mathrm{~nm}$, which is a well suited range to expect energy transfer.

We performed PL measurements to obtain the relative fluorescence intensity of the $\mathrm{CD}$ and the fluorescein and compared that to the PL of the combined $\mathrm{CD} / \mathrm{F}$ system (Figure 4a). For an excitation at $360 \mathrm{~nm}$, a decrease of the CD fluorescence emission at around $440-450 \mathrm{~nm}$ is observed in the presence of the acceptor dye, which is a qualitative confirmation for FRET (PL excitation spectra for only CDs and for increasing amounts of fluorescein are further shown in the Supporting Information, Figure S4). At the same time, a strong increase of the $\mathrm{PL}$ of the $\mathrm{CD} / \mathrm{F}$ conjugate, along with a small red-shift of the PL maximum from 525 to $532 \mathrm{~nm}$, is observed. The enhanced fluorescence is an indication for energy "feeding" from the donor CDs to the acceptor dyes. However, in addition to energy transfer, electrostatic interactions between anionic fluorescein and cationic CDs can also have an influence. It has been reported that fluorescein shows enhanced $\mathrm{PL}^{36}$ along with a red-shift in the fluorescence and absorption spectra ${ }^{37}$ in the presence of cationic surfactants. This change of spectral properties has been explained by a shift of the chemical equilibrium between monoanionic and dianionic fluorescein in solution due to electrostatic stabilization. ${ }^{31,36}$ Given the positive surface charge of the CDs, such electrostatic effects should also be expected to occur in the $\mathrm{CD} / \mathrm{F}$ vesicles.

We performed PL excitation (PLE) measurements to analyze the interaction between $\mathrm{CDs}$ and fluorescein in more detail. As shown in Figure $4 \mathrm{~b}$, the emission of the $\mathrm{CD} / \mathrm{F}$ sample at $554 \mathrm{~nm}$ is increased compared to a vesicle sample with only fluorescein (F) for an excitation between 280 and $530 \mathrm{~nm}$ (Figure $4 \mathrm{~b}$ ). The onset of CD absorption is at $450 \mathrm{~nm}$ (Figure 2). Below that wavelength, the increased PL excitation of the $\mathrm{CD} / \mathrm{F}$ sample can be explained since energy is transferred from the CDs to the dye. However, the PLE of the $\mathrm{CD} / \mathrm{F}$ conjugate cannot be reproduced by adding the $\mathrm{CD}$ absorption to the fluorescein PLE according to $P L E_{F}+a \cdot a b s_{C D}$ $=P L E_{C D / F}$ (with $a$ being a weighting factor). Furthermore, the $\mathrm{CD} / \mathrm{F}$ excitation between 450 and $530 \mathrm{~nm}$ also increases, a)

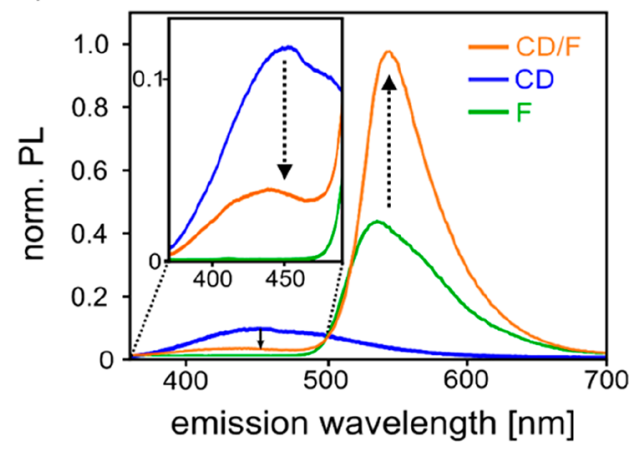

b)

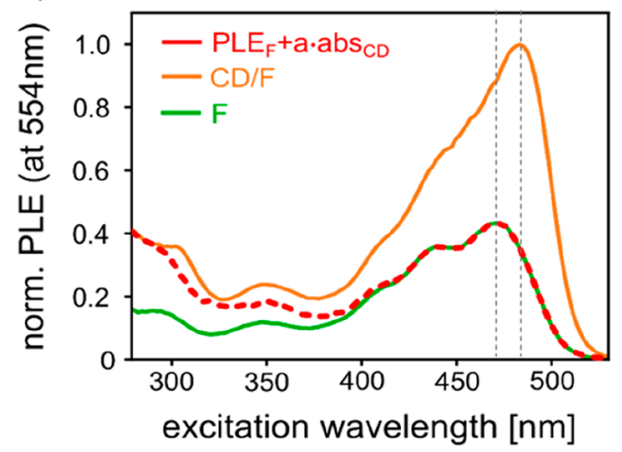

Figure 4. Normalized PL and photoluminescence excitation (PLE) spectra: (a) The PL spectrum of the $\mathrm{CD} / \mathrm{F}$ system (orange) after excitation with $360 \mathrm{~nm}$. A decrease of the CD fluorescence emission at $450 \mathrm{~nm}$ is observed, while the fluorescein fluorescence emission shows an increase at the same time. This result confirms the occurrence of FRET. (b) Normalized PLE spectra of the CD/F (orange line) and fluorescein vesicles detected at $554 \mathrm{~nm}$. The red dotted line represents the sum of the fluorescein PLE (green line) and the weighted absorption $a \cdot a b s_{C D}$ of the CDs for $a=0.25$. Compared to the PLE spectrum of fluorescein in vesicles, the excitation maximum of the $\mathrm{CD} / \mathrm{F}$ sample is shifted from 472 to $484 \mathrm{~nm}$ (dotted lines).

although the $\mathrm{CD}$ absorption is negligible in this range and the main PL excitation is shifted by $\sim 12 \mathrm{~nm}$. This shift is concordant with the reported red-shift in the absorption 

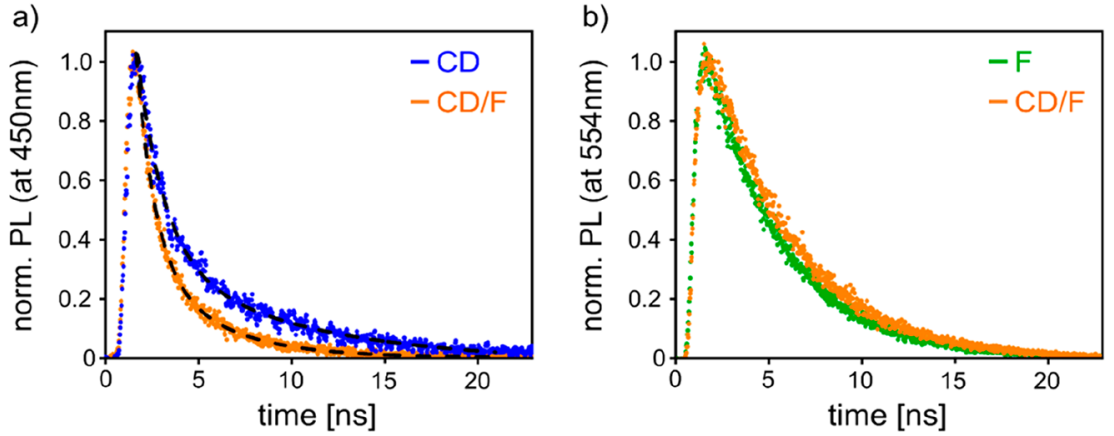

Figure 5. Time-resolved PL decays: (a) Donor fluorescence decay traces of the CD (blue) and the CD/F conjugate vesicles (orange). The dotted black lines represent the biexponential fits. (b) Acceptor fluorescence decay traces of the $\mathrm{F}$ (green) and the $\mathrm{CD} / \mathrm{F}$ sample (orange). In all cases, the samples are excited at $360 \mathrm{~nm}$.

spectra for the transition from monoanionic to dianionic fluorescein. ${ }^{31,36}$ Together, both observations are indicative for the stabilization of dianionic fluorescein in the presence of the CDs, which is also evidence for ionic interactions between fluorescein and the CDs in the bilayer membrane.

Due to the ionic effects, the efficiency of energy transfer between the CDs and the fluorescein cannot be determined by only using the steady state fluorescence intensity of CDs in absence and presence of the dye. We therefore performed PL lifetime measurements to analyze the rate of energy transfer in more detail. Fluorescence decay traces of the $\mathrm{CD}$ and the $\mathrm{CD} /$ $\mathrm{F}$ conjugate are displayed in Figure 5a. In presence of fluorescein, an acceleration of the fluorescence decay at 450 $\mathrm{nm}$ for an excitation at $360 \mathrm{~nm}$ is visible. We find that the PL of the $\mathrm{CD}$ and the $\mathrm{CD} / \mathrm{F}$ samples displays a nonmonoexponential decay (Figure 5a). Indeed, a multiexponential decay of PL intensity has been reported for CDs, which has been attributed to several sources, such as different emissive substructures or molecular configurations within the CDs. $^{20,21,38,39}$ The intensity decay curves shown in Figure 5a are reproduced by using a biexponential decay model according to

$$
I(t)=\sum_{i=1}^{n} \alpha_{i} \mathrm{e}^{-t / \tau_{i}}
$$

with $\alpha_{i}$ being the amplitude, $\tau_{i}$ the respective decay time, and $n$ $=2$. For the CD sample, we obtain a short decay component of 1.5 ns and a long decay time component of 8.4 ns. For the $\mathrm{CD} / \mathrm{F}$ conjugate, the decay lifetimes are reduced to 1.0 and 3.7 $\mathrm{ns}$, respectively. An overview of the individual decay times and corresponding amplitudes is given in Table 1 . Since both fluorescence decay components of the CDs are accelerated in the presence of the fluorescein, we use the amplitude-weighted average decay times $\left\langle\tau_{C D}\right\rangle$ and $\left\langle\tau_{C D / F}\right\rangle$ to calculate the energy transfer efficiency $E$ and the energy transfer rate $\kappa_{E T}$ via $E=1-\frac{\left\langle\tau_{C D / F}\right\rangle}{\left\langle\tau_{C D}\right\rangle}$ and $k_{E T}=\frac{1}{\left\langle\tau_{C D / F}\right\rangle}-\frac{1}{\left\langle\tau_{C D}\right\rangle}{ }^{40}$ and obtain a transfer rate of $k_{E T}=0.27 \mathrm{~ns}^{-1}$ and a FRET efficiency of $51.3 \%$.

The use of these equations to calculate the rate and efficiency is justified by the configuration of the $\mathrm{CD} / \mathrm{F}$ conjugate within the bilayer. The separation distance between CDs and fluorescein is fixed since the hydrophobic CDs are located between the bilayer sheets and the fluorescein is attached to the lipid heads. In addition, the fluorescein lipids are electrostatically attracted to the CDs due to their opposite charge. Both donor and acceptor are therefore not spatially
Table 1. Decay Times and Normalized Amplitudes for CD (360/450) and CD/F (360/450) Were Determined by a Biexponential Fit to the Intensity Decay Traces ${ }^{a}$

$\begin{array}{lcccccc} & \lambda_{\text {exc }} / \lambda_{\text {em }}(\mathrm{nm}) & \tau_{i}(\mathrm{~ns}) & \alpha_{i} & \left\langle\tau_{i}\right\rangle(\mathrm{ns}) & E(\%) & k_{E T}\left(\mathrm{~ns}^{-1}\right) \\ \mathrm{CD} & 360 / 450 & 1.5 & 0.65 & 3.9 & 51.3 & 0.27 \\ & & 8.4 & 0.35 & & & \\ \mathrm{CD} / \mathrm{F} & 360 / 450 & 1.0 & 0.65 & 1.9 & & \\ & & 3.7 & 0.35 & & & \\ \mathrm{~F} & 360 / 554 & 4.2 & & & & \\ \mathrm{CD} / \mathrm{F} & 360 / 554 & 4.8 & & & & \end{array}$

${ }^{a}$ Furthermore, the amplitude-weighted average decay times of the CDs in presence and absence of fluorescein are listed as well as the resulting energy transfer efficiency and rate. Decay times for F (360/ $554)$ and $\mathrm{CD} / \mathrm{F}(360 / 554)$ were determined by a mono-exponential fit to the fluorescence decay traces.

distributed across the fluid membrane, which would require a more complex analysis. ${ }^{41}$

The obtained energy transfer efficiency of $51.3 \%$ suggests that the separation distance between the CDs and fluorescein is close to the Förster distance $R_{0}$, where the transfer efficiency is $50 \%$ by definition. One can estimate the Förster distance of the CD/F FRET pair in a membrane environment from the obtained data. We assume a separation distance $r=4 \mathrm{~nm}$ between donor and acceptor, which corresponds to approximately half the width of a DOPC bilayer membrane $(\sim 6 \mathrm{~nm})$ and the radius of the CDs. With the transfer rate $k_{E T}, R_{0}$ can now be calculated according to $k_{E T}=\frac{1}{\left\langle\tau_{C D}\right\rangle}\left(\frac{R_{0}}{r}\right)^{6}$. The resulting Förster distance $R_{0}$ of $\sim 4 \mathrm{~nm}$ is in good agreement with reported literature values. ${ }^{10,33,34,40}$ Furthermore, in control measurements where the fluorescein is free in solution, we find a lower FRET efficiency of only $17.1 \%$. This can be explained by a larger CD-dye separation distance since the fluorescein is no longer attached to the membrane (Supporting Information, Figure S5).

Aside from energy transfer from the CDs, the fluorescein molecules can also be directly excited with UV light. Compared to the accelerated donor fluorescence decay in the $\mathrm{CD} / \mathrm{F}$ sample, the intensity decay of the fluorescein acceptor at $554 \mathrm{~nm}$ is delayed (Figure $5 \mathrm{~b}$ ). The PL decay curves of the $\mathrm{CD} / \mathrm{F}$ and the fluorescein system for an excitation at $360 \mathrm{~nm}$ can be reproduced with a monoexponential fit, and a lifetime increase from 4.2 to $4.8 \mathrm{~ns}$ is observed. This prolonged fluorescence lifetime, however, may not only be the result of energy "feeding" from the donor CDs 
to the acceptor fluorophores but is also the consequence of ionic interactions and the stabilization of the fluorescein dianion in the vesicle membrane. ${ }^{36}$

In conclusion, we reported an approach to introduce blue emitting CDs between the leaflets of a fluorescein labeled lipid bilayer membrane. The spectral overlap between both fluorescent components allows establishing trans-membrane energy transfer between the bilayer leaflets. In addition, ionic interactions between the positively charged $\mathrm{CDs}$ and the anionic dye molecules result in fluorescence enhancement and localization of the acceptor molecules next to the energyfeeding donor. Improving the fluorescence emission of membrane bound dyes is advantageous for membrane imaging and microscopy studies of bilayer systems. Furthermore, any changes of the membrane potential via trans-membrane ion transport or due to an externally applied electric field would affect the strength of the electrostatic attraction between dyes and CDs. This has an influence on both the FRET efficiency as well as on ionic effects. Overall, the simultaneous balancing of both energy transfer and electrostatic interactions within a model membrane, as shown here, represents a general pathway to control and investigate membrane related processes with unprecedented spatiotemporal control and resolution.

\section{ASSOCIATED CONTENT}

\section{S Supporting Information}

The Supporting Information is available free of charge on the ACS Publications website at DOI: 10.1021/acs.nanolett.9b01071.

Additional details on sample preparation and characterization. Details on microscopy and spectroscopy methods. Control measurements with free fluorescein in solution (PDF)

\section{AUTHOR INFORMATION}

\section{Corresponding Authors}

*E-mail: t.lohmueller@lmu.de.

*E-mail: mahuergo@inifta.unlp.edu.ar.

*E-mail: feldmann@lmu.de.

\section{ORCID}

Fernando Pschunder: 0000-0003-1196-6524

Santanu Bhattacharyya: 0000-0001-5572-2551

Theobald Lohmüller: 0000-0003-2699-7067

\section{Present Address}

\#(S.B.) Department of Chemical Sciences, IISER Berhampur, Transit campus (Govt. ITI), Eng. School Road, Berhampur, Odisha 760010, India.

\section{Notes}

The authors declare no competing financial interest.

\section{ACKNOWLEDGMENTS}

Financial support by the ERC through the Advanced Investigator Grant HYMEM, by the DFG through SFB 1032, project A08, and by the Bayerisches Hochschulzentrum Lateinamerika through BAYLAT is gratefully acknowledged. This work has been financially supported by ANPCyT (PICT 2016-0679), CONICET, and UNLP. The Alexander von Humboldt-Foundation supported S.B. The authors want to thank Dr. E. D. Prieto for AFM technical support and analysis and A. Richter for fruitful discussions.

\section{REFERENCES}

(1) Bezanilla, F. Nat. Rev. Mol. Cell Biol. 2008, 9, 323.

(2) Neher, E.; Sakmann, B. Sci. Am. 1992, 266 (3), 44-51.

(3) Brüggemann, A.; George, M.; Klau, M.; Beckler, M.; Steindl, J.; Behrends, J. C.; Fertig, N. HEKA Impulse 2005, 03, 2.

(4) Miller, E. W. Curr. Opin. Chem. Biol. 2016, 33, 74-80.

(5) González, J. E.; Tsien, R. Y. Biophys. J. 1995, 69 (4), 1272-1280.

(6) Azzi, A. Q. Q. Rev. Biophys. 1975, 8 (2), 237-316.

(7) Zal, T.; Gascoigne, N. R. J. Biophys. J. 2004, 86 (6), 3923-3939.

(8) Johnstone, R. M.; Laris, P. C.; Eddy, A. A. J. Cell. Physiol. 1982, 112 (2), 298-301.

(9) Sapsford, K. E.; Berti, L.; Medintz, I. L. Angew. Chem., Int. Ed. 2006, 45 (28), 4562-4589.

(10) Haugland, R. P. Molecular Probes Handbook - A Guide to Fluorescent Probes and Labeling Technologies, 11th ed.; Invitrogen: 2010.

(11) Resch-Genger, U.; Grabolle, M.; Cavaliere-Jaricot, S.; Nitschke, R.; Nann, T. Nat. Methods 2008, 5, 763.

(12) Kloepfer, J. A.; Cohen, N.; Nadeau, J. L. J. Phys. Chem. B 2004, 108 (44), 17042-17049.

(13) Marshall, J. D.; Schnitzer, M. J. ACS Nano 2013, 7 (5), 46014609.

(14) Park, K.; Kuo, Y.; Shvadchak, V.; Ingargiola, A.; Dai, X.; Hsiung, L.; Kim, W.; Zhou, Z. H.; Zou, P.; Levine, A. J.; Li, J.; Weiss, S. Sci. Adv. 2018, 4 (1), e1601453.

(15) Park, K.; Weiss, S. Biophys. J. 2017, 112 (4), 703-713.

(16) Zheng, W.; Liu, Y.; West, A.; Schuler, E. E.; Yehl, K.; Dyer, R. B.; Kindt, J. T.; Salaita, K. J. Am. Chem. Soc. 2014, 136 (5), 19921999.

(17) Hardman, R. Environ. Health Perspect. 2006, 114 (2), 165-172.

(18) Petryayeva, E.; Algar, W. R.; Medintz, I. L. Appl. Spectrosc. 2013, 67 (3), 215-252.

(19) Das, R.; Bandyopadhyay, R.; Pramanik, P. Mater. Today Chem. 2018, 8, 96-109.

(20) Fu, M.; Ehrat, F.; Wang, Y.; Milowska, K. Z.; Reckmeier, C.; Rogach, A. L.; Stolarczyk, J. K.; Urban, A. S.; Feldmann, J. Nano Lett. 2015, 15 (9), 6030-6035.

(21) Ehrat, F.; Bhattacharyya, S.; Schneider, J.; Löf, A.; Wyrwich, R.; Rogach, A. L.; Stolarczyk, J. K.; Urban, A. S.; Feldmann, J. Nano Lett. 2017, 17 (12), 7710-7716.

(22) Jelinek, R. Carbon Quantum Dots, 1st ed.; Springer International Publishing: Cham, 2017; p V, 130.

(23) Roy, P.; Chen, P.-C.; Periasamy, A. P.; Chen, Y.-N.; Chang, H.T. Mater. Today 2015, 18 (8), 447-458.

(24) Cao, L.; Wang, X.; Meziani, M. J.; Lu, F.; Wang, H.; Luo, P. G.; Lin, Y.; Harruff, B. A.; Veca, L. M.; Murray, D.; Xie, S.-Y.; Sun, Y.-P. J. Am. Chem. Soc. 2007, 129 (37), 11318-11319.

(25) Jiang, K.; Sun, S.; Zhang, L.; Lu, Y.; Wu, A.; Cai, C.; Lin, H. Angew. Chem., Int. Ed. 2015, 54 (18), 5360-5363.

(26) Yang, S.-T.; Cao, L.; Luo, P. G.; Lu, F.; Wang, X.; Wang, H.; Meziani, M. J.; Liu, Y.; Qi, G.; Sun, Y.-P. J. Am. Chem. Soc. 2009, 131 (32), 11308-11309.

(27) Nandi, S.; Malishev, R.; Parambath Kootery, K.; Mirsky, Y.; Kolusheva, S.; Jelinek, R. Chem. Commun. 2014, 50 (71), 1029910302.

(28) Nandi, S.; Malishev, R.; Bhunia, S. K.; Kolusheva, S.; Jopp, J.; Jelinek, R. Biophys. J. 2016, 110 (9), 2016-2025.

(29) Cheng, F.; An, X.; Zheng, C.; Cao, S. RSC Adv. 2015, 5 (113), 93360-93363.

(30) Clegg, R. M. Curr. Opin. Biotechnol. 1995, 6 (1), 103-110.

(31) Kibblewhite, J.; Drummond, C. J.; Grieser, F.; Thistlethwaite, P. J. J. Phys. Chem. 1989, 93 (21), 7464-7473.

(32) Almeida, P. F. F.; Vaz, W. L. C. Lateral Diffusion in Membranes. In Handbook of Biological Physics - Structure and Dynamics of Membranes: From Cells to Vesicles, 1st ed.; Sackmann, R. L. a. E., Ed.; Elsevier Science B.V: 1995; Vol. 1.

(33) Wu, P. G.; Brand, L. Anal. Biochem. 1994, 218 (1), 1-13.

(34) Pietraszewska-Bogiel, A.; Gadella, T. W. J. J. Microsc. 2011, 241 (2), 111-118. 
(35) Tristram-Nagle, S.; Petrache, H. I.; Nagle, J. F. Biophys. J. 1998, 75 (2), 917-925.

(36) Song, A.; Zhang, J.; Zhang, M.; Shen, T.; Tang, J. a. Colloids Surf., A 2000, 167 (3), 253-262.

(37) Hadjianestis, J.; Nikokavouras, J. J. Photochem. Photobiol., A 1993, 69 (3), 337-343.

(38) Röding, M.; Bradley, S. J.; Nydén, M.; Nann, T. J. Phys. Chem. C 2014, 118 (51), 30282-30290.

(39) Bao, L.; Zhang, Z.-L.; Tian, Z.-Q.; Zhang, L.; Liu, C.; Lin, Y.; Qi, B.; Pang, D.-W. Adv. Mater. 2011, 23 (48), 5801-5806.

(40) Lakowicz, J. R. Principles of Fluorescence Spectroscopy, 3rd ed.; Springer US: 2006.

(41) Loura, L. M. S.; Prieto, M. Front. Psychol. 2011, 2, 82. 